\title{
Systematic review of resilience-enhancing, universal, primary school-based mental health promotion programs
}

\author{
Amanda Fenwick-Smith, Emma E. Dahlberg and Sandra C. Thompson * (B)
}

\begin{abstract}
Background: Wellbeing and resilience are essential in preventing and reducing the severity of mental health problems. Equipping children with coping skills and protective behavior can help them react positively to change and obstacles in life, allowing greater mental, social and academic success. This systematic review studies the implementation and evaluation of universal, resilience-focused mental health promotion programs based in primary schools.

Methods: A systematic review of literature used five primary databases: PsycINFO; Web of Science; PubMed; Medline; Embase and The Cochrane Library; and keywords related to (a) health education, health promotion, mental health, mental health promotion, social and emotional wellbeing; (b) school health service, student, schools, whole-school; (c) adolescent, child, school child, pre-adolescent; (d) emotional intelligence, coping behavior, emotional adjustment, resilienc*, problem solving, to identify relevant articles. Articles included featured programs that were universally implemented in a primary school setting and focused on teaching of skills, including coping skills, help-seeking behaviors, stress management, and mindfulness, and were aimed at the overall goal of increasing resilience among students.

Results: Of 3087 peer-reviewed articles initially identified, 475 articles were further evaluated with 11 reports on evaluations of 7 school-based mental health promotion programs meeting the inclusion criteria. Evaluation tools used in program evaluation are also reviewed, with successful trends in evaluations discussed. Encouraging results were seen when the program was delivered by teachers within the schools. Length of programing did not seem important to outcomes. Across all 7 programs, few long-term sustained effects were recorded following program completion.
\end{abstract}

Conclusions: This review provides evidence that mental health promotion programs that focus on resilience and coping skills have positive impacts on the students' ability to manage daily stressors.

Keywords: Mental health, Health promotion, Primary school, Resilience, Universal intervention, Child

\section{Background}

This review looks at resilience-boosting mental health promotion programs implemented universally at schools for primary school-aged children (5-12 years). Wellbeing and resilience are important in preventing and reducing the severity of mental health problems. The skills of problem solving, building and maintaining interpersonal relationships, and realistic goal-setting are well-established as

\footnotetext{
* Correspondence: sandra.thompson@uwa.edu.au

Western Australian Centre for Rural Health, University of Western Australia, 167 Fitzgerald St, Geraldton, WA 6531, Australia
}

enhancing an individual's ability to contribute meaningfully in daily life. There is substantial literature on resilience [1] which is defined as a capacity or set of skills that allows a person to "prevent, minimize or overcome the damaging effects of adversity" [2] and includes factors that are internal and external to the person - emotions, behavior, biology, development, and context affect mental health [3]. Potential risks for poor self-esteem and mental health can be overcome by protective factors, including one's coping skills, healthy family and social relationships, help-seeking behaviors, and meaningful activities in interactions [4].

(c) The Author(s). 2018 Open Access This article is distributed under the terms of the Creative Commons Attribution 4.0 International License (http://creativecommons.org/licenses/by/4.0/), which permits unrestricted use, distribution, and 
Resilience theory states that all children, regardless of risk or current mental health status, can benefit from help and support in the development of effective, mentally-healthy strategies and resilience skills [5]. Support for and a focus on the development of children's resilience skills does not lead to a risk-free life, but can increase a child's ability to seek support while building their self-worth and self-efficacy. By providing children with skills with which to cope with negative life stressors through the promotion of resilience and protective factors, children can thrive despite obstacles [6]. An argument for a population approach for mental health strengthening can be extrapolated from Geoffrey Rose's argument that the largest number of cases of ill health happen not in those at high risk, but in those who have just some risk, simply because in a normal population distribution more people (and hence adverse events) will occur to them [7]. Since all people experience adversity at some point in their life, teaching strategies for resilient thinking would be better applied in advance to the potential "at risk" population. The positive outcomes and possibilities associated with strengthening children's resilience universally applied can act as a mitigating approach, allowing for early support and strengthening of mental health, rather than requiring interventions for acute situations in the future [8]. The approach of boosting resilience can enhance children's abilities to self-protect, as well as being an effective counter to offset the effects of maltreatment and potential traumatic life events [9-11]. As such, universal application of programs to enhance resilience stands as not only useful for those recognized as being at risk and who require additional mental health support currently, but also as a protective shield for all children moving through life.

\section{Universal, school-based programing}

School-based mental health promotion programs delivered to all students within a class, grade, or the entire school are categorized as universal programs. In developed countries, all children are required to attend school, making it an ideal setting for programs providing key interventions for children, particularly children from challenged families, homes and communities that may not have easy access to community or home-based intervention programs [12]. Mental health promotion programs have been developed and implemented in schools using a variety of different approaches. Many mental illness prevention or intervention programs use a targeted approach, focusing on children deemed at risk due to their background, history or signs of mental health problems, usually based upon defined socio-demographic factors or certain behavioral characteristics.
Universal programs vary in their approach and implementation. Some universal programs are class-based, with weekly sessions delivered by classroom teachers or program staff to the entire classroom. Another universal approach is to change the entire school environment to be friendlier and more supportive of positive mental health messages, and this is often implemented in combination with class-based approaches [13]. Class-based, universal mental health promotion programs vary in their aims, focusing on different elements of cognitive or affective skills and behaviors, environmental or cultural factors, while increasing knowledge of mental health and resources.

Mental health promotion programs specifically targeting resilience may be referred to as social and emotional learning programs, mindfulness programs, stress management programs, or emotional wellbeing programs and vary in terms of curriculum, length and implementation, and use of different tools and activities to convey key themes and topics. Methods of delivery vary as well, including the use of clinical tools, educational resources, training of teachers and parents, changes to school systems and resources, and use of narrative tools. As such, the curricula used in these programs vary, although all utilize a pre-established definition of resilience and the desired outcomes to be achieved from a social and emotional learning program. The most effective social and emotional learning curricula are highly interactive and use a variety of educational tools, addressing both specific and general skills, and are delivered in supportive environments [14]. Mental health promotion programs promoting resilience focus on the development of coping skills, mindfulness, emotion recognition and management, empathic relationships, self-awareness and efficacy, and help-seeking behavior. Secondary outcomes often include decreased symptoms of anxiety, depression, and increased academic outcomes.

\section{Relevant research reviews}

Given the importance and reach of school settings, many reports describe universal, school-based mental health promotion programs. Prior reviews have explored school-based mental health promotion programs in different contexts, countries, applications, and within specific demographic parameters. There are many reviews addressing targeted programs aimed at suicide prevention, sexual health, substance abuse and misuse, physical activity and nutrition improvement and these often measure as secondary outcomes changes in self-efficacy, coping and resilience skills [5, 15-17]. A number of reviews analyzing mental health promotion programs that focus on resilience across a range of age groups have established that school-based interventions can have significant impacts on achievement, 
social and emotional skills, behavior, and symptoms of anxiety and depressive disorders [12, 16, 18]. In their 2017 review, Dray and colleagues looked at control-based trial evaluations of programs of universal resilience-programing in schools spanning all ages, reporting on those that yielded significant results in resilience factor changes. Durlak and colleagues compared 213 programs, also targeting all age groups, assessing the outcomes on attitudes, behaviors and academic performance and analyzing effect size and factors that moderate program outcomes. Waere and Mind assessed the key features that make school-based curricula successful as an approach, highlighting the importance of social and emotional competence as part of the curriculum within schools [12]. Another review considered studies on mental health promotion programs solely conducted with control and comparison groups [19].

\section{The current review}

This review aims to inform policy, programing and evaluation of universal, resilience-focused mental health interventions for primary school-aged children as it focuses on the specific tools and key elements for the population that will benefit the most from increased resilience in an easy-to-reach setting, aspects which have not been highlighted in previous reviews. The multitude of existing mental health promotion programs highlights the need to establish what specific elements and evaluations contribute to successful programing. Unlike previous reviews, this review focuses on programs delivered solely to primary school students (aged 5-12 years), as there is evidence that the younger the implementation of mental health promotion and resilience programing, the greater the positive effect $[3,20$, 21]. Rather than focusing on the program curriculum, it considers the criteria for implementation and key elements of programing for a comprehensive intervention, highlighting the elements of that allow for best program fidelity and student engagement. It also describes the criteria and outcome measures (tools and methods) used in implementing and evaluating resilience-focused, universal school-based mental health promotion programs.

\section{Methods}

Studies eligible for inclusion were published from 2002 to 2017, describe mental health promotion programs focusing on resilience and protective factors, and were delivered universally at schools for primary school children aged 5-12 years. A universal program is defined as being a program offered for a specific all-inclusive group, whether it be the entire school, grade or classroom. All students within the group participate in at least one component of the program, regardless of their mental health status and risk factors. Resilience is defined as a capacity or set of skills that allows a person to "prevent, minimize or overcome the damaging effects of adversity" [2], through the promotion of protective factors including coping skills, peer socialization and empathy building, self-efficacy, help-seeking behaviors, mindfulness and emotion literacy.

\section{Search procedures}

A preliminary review of literature revealed key terms related to resilience-focused, school-based, universal mental health promotion programs. A broad search strategy was then developed to identify relevant peer-reviewed articles in five primary databases: PsycINFO; Web of Science; PubMed; Medline; Embase and The Cochrane Library. The search strategy was modified as necessary for advanced searches of each database, using keyword search criteria: (a) health education, health promotion, mental health, mental health promotion, social and emotional wellbeing; (b) school health service, student, schools, whole-school; (c) adolescen", child, school child, pre-adolescent; (d) emotional intelligence, coping behavior, emotional adjustment, resilienc*, problem solving. Searches were conducted in September 2016 and updated in May 2018. Articles were initially screened by abstract by the lead author. All full-text articles were reviewed by two reviewers, with additional checks and consultations with other authors, to ensure consensus around those articles where eligibility was less clear. Snowball citation was used to identify other relevant articles.

\section{Inclusion criteria}

To be included in the review, each study had to meet the following criteria: (a) adhere to the above definition of a universal program; (b) be based in a primary school; (c) be delivered to children aged between 5 and 12 years of age; (d) focus on resilience and protective factors (meeting the above definition); (e) contain a qualitative, quantitative or mixed-methods evaluation of the program; (f) be published in English since 2002 in a peer-reviewed journal.

\section{Exclusion criteria}

Programs targeting specific behaviors where resilience is a secondary outcome, or programs primarily focusing on post-traumatic stress among students affected by natural disasters or war were not included. Programs with the ultimate goal and outcome measurements relating to a specific behavior, emotional condition or mental illness were not included, even if the tools taught in the intervention could be classified as resilience promoting. 
Universal programs that sought to change school atmosphere through teacher resilience training, or increasing school health services were not included. After-school or recess resilience programing was not included, even if it took place at a school. Programs that were available but not implemented universally were not included, as the self-selecting nature of optional programing is unlikely to reach the most at-risk children, and such programs do not insure a comprehensive program for all students regardless of risk. Studies where many students were outside of the age group and during a transition period between different schools were not included. Resilience programing that fits our inclusion criteria but is solely delivered to a population that has been exposed to high stress situations and is at risk or may develop PTSD are not included. Unpublished dissertations, grey literature and reports were not included.

\section{Excluded studies}

It is worth commenting upon how exclusion criteria were applied in practice. A number of programs were not included in this review despite having a resilience focus, being universally-delivered and school-based because they have not been reported upon within the preceding 15 years (since 2002). Other excluded programs had an ultimate goal that was not general mental health promotion program, but rather aimed at addressing a specific condition or behavior through the promotion of certain resilience skills and protective factors. Notable programs include the Penn Resilience Program, which has been shown to reduce depressive symptoms through the cognitive-behavioral therapy programing, including the promotion of coping skills [22]. The Good Behavior Games specifically target behavior control through the promotion of resilience, but fall outside of the age range of this review [23]. REACH for RESILIENCE promotes resilience skills to prevent anxiety problems, and targets very young children [24]. The nation-wide Australian program, beyondblue, focuses on social and resilience skills to prevent depression, targeting adolescents [25]. Evaluations of the FRIENDS program were not included as it targets childhood anxiety through the promotion of social-emotional skills [26]. Another exclusion was the Aussie Optimism: Positive Thinking Skills Program (AOP-PTS) which promotes social and coping skills to prevent and address depression symptoms [27].

\section{Article quality assessment}

The Mixed Methods Appraisal Tool (MMAT) was used to assess the quality of included studies and provides a validated method of assessing qualitative, quantitative, and mixed methods studies. After the initial screening, articles were scored based on the criteria for each respective study [28]. Two researchers independently assessed each article [29]. Of note, the tool does not address the quality of the reporting, but only the quality of the reported methods of the study.

\section{Results}

The initial search strategy shows that of an initial 3087 publications identified using the search terms and following abstract assessment of 475 references, 34 articles were selected for full-text assessment. An additional 7 articles were identified through citation snowballing and after reading of the full-text so that 41 articles were fully assessed for eligibility. A total of 11 studies reporting on 7 programs met all the inclusion criteria (Fig. 1), with key characteristics including MMAT scores recorded (Table 1). The most common reasons for exclusion were: focus on trauma, incorrect age group or target population; not meeting our definition of universal programs; and lack of focus on resilience and protective factors. Included articles. Key elements of each program's curriculum and implementation are shown (Table 2).

\section{Aim of the program}

The aims of the seven programs (reported on in eleven articles) included varied in their approach to resilience and the protective factors they sought to address. All six programs sought to increase social and emotional competencies with the ultimate aim of increasing mental wellbeing and future protection from risks. Six articles, addressing 2 different programs, Mindfulness-Based Stress Reduction and Zippy's Friends, specifically sought to improve psychological functioning with the goal of ameliorating the negative effects of stress and increasing coping skills [30-35]. The RALLY program aimed at increasing the prevalence of resilience protective factors in students, with a particular focus on academic outcomes and learning potential [36] while the Up program, a social and emotional competencies program, aimed at enhancing existing competencies and decreasing inequity in social and emotional competencies across socioeconomic lines [37]. The You Can Do It! (YCDI!) Education program sought to ameliorate children's ability to positively control their emotions in daily life [38]. All programs sought to improve the outcomes of one or more protective factors, hypothesizing increased resilience as a result. A strong emphasis on increased coping skills and strategies as well as improved relationships was evident in all the programs.

\section{Target population}

Universal programs demand the application of the program to an entire cohort of students, but how that was done varied from delivering the program to 


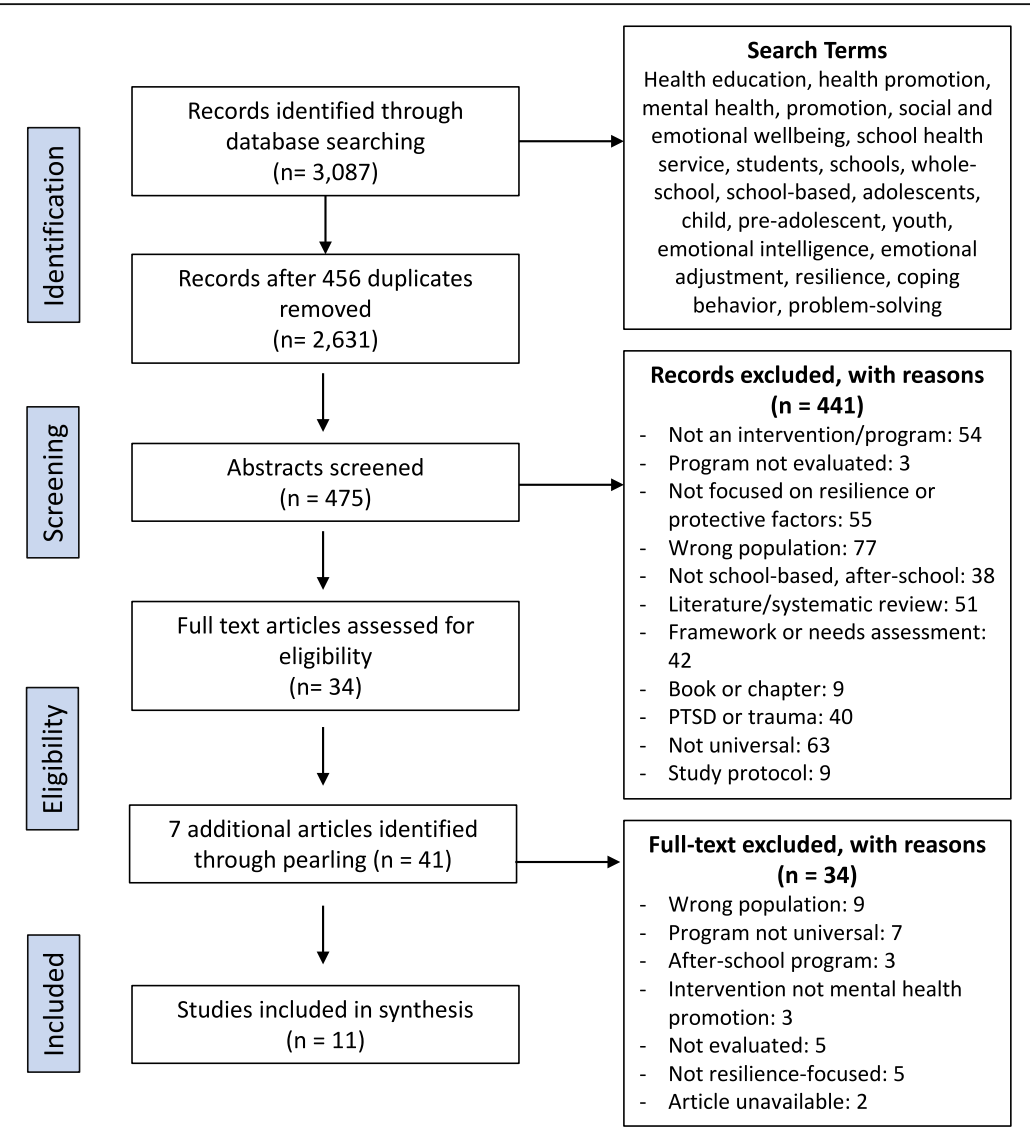

Fig. 1 Flow diagram of selection process for relevant literature

an entire class, across an entire grade or across multiple schools. As such, sample size varied significantly between studies. Details of sample populations (Table 1) show all but two studies were implemented and evaluated across multiple schools, with ten of eleven conducted across multiple classrooms [30-35, 37-39]. Age groups varied across the programs, with 4 studies addressing populations 10 years and above [30, 36, 38, 39], and 6 studies addressing populations younger than 10 years of age [31-35, 40]. Socio-demographic profiles of students varied across studies. Four studies described programs delivered at socio-economically disadvantaged schools $[30,31,33,36]$ whereas four programs took place in middle or upper class neighborhoods [32, 37, 39, 40]. Dufour et al. (2011) did not report on socio-demographic data of students who received the program [34] whereas the students involved in the report by Holen et al (2012) were from homes where parents had educational attainment levels higher than the national average [35]. Yamamoto et al. (2017) delivered the program to students in the Tokyo Metropolitan Area, making no demographic distinctions, other than to address the specific contextual implications of Japanese emotion- and stress-culture as impactful in their student population [38].

\section{Key elements of programs}

Key elements of the programs (Table 2) show that Malti et al (2008) was the only study in which the program comprised more than one student-focused component [36]. Although only a few components were delivered universally, all students were exposed to at least one component of the program [36]. The Up program included parent and teacher training, and school environment programing [37] and program fidelity and adaptability were identified as key contributing factors to successful implementation with four studies reporting high levels of program fidelity and program support $[32,33,39,40]$. The five studies that implemented and evaluated the Zippy's Friends program described no changes in curriculum or delivery, but allowed for activity adaptability during sessions [31-35]. Teachers delivering it felt equipped to adapt the program as they saw necessary to their class while still maintaining high program fidelity [34]. Adaptability was also highlighted as being an important program factor for the You Can 
Table 1 Summary of Articles Included in the Review

\begin{tabular}{|c|c|c|c|c|c|c|}
\hline First author, year published Study type & $\begin{array}{l}\text { Program } \\
\text { Name }\end{array}$ & Location & Study Type & Sample Size & Aim of Program and Study & $\begin{array}{l}\text { MMAT } \\
\text { Score }\end{array}$ \\
\hline $\begin{array}{l}\text { Malti (2008)[36] } \\
\text { Program Evaluation: Relationships } \\
\text { as key to student development }\end{array}$ & RALLY & $\begin{array}{l}\text { United } \\
\text { States }\end{array}$ & $\begin{array}{l}\text { Quasi- } \\
\text { experimental, } \\
\text { Mixed methods }\end{array}$ & 92 students & $\begin{array}{l}\text { - Improved resilience } \\
\text { outcomes, learning interest } \\
\text { and decrease risk-taking. } \\
\text { - Assess program } \\
\text { implementation quality }\end{array}$ & $100 \%$ \\
\hline $\begin{array}{l}\text { Sibinga (2016)[30] } \\
\text { School-Based Mindfulness Instruction: An } \\
\text { RCT }\end{array}$ & $\begin{array}{l}\text { Mindfulness- } \\
\text { Based Stress } \\
\text { Reduction } \\
\text { (MBSR) }\end{array}$ & $\begin{array}{l}\text { United } \\
\text { States } \\
\text { (Baltimore, } \\
\text { Maryland) }\end{array}$ & $\begin{array}{l}\text { Randomized, } \\
\text { Active } \\
\text { Controlled Trial }\end{array}$ & $\begin{array}{l}\text { Interv: } 159 \\
\text { students }\end{array}$ & $\begin{array}{l}\text { - Improve psychological } \\
\text { functioning to decrease } \\
\text { negative effects of stress } \\
\text { - Reduce worries about future }\end{array}$ & $50 \%$ \\
\hline $\begin{array}{l}\text { Kraag (2009)[39] } \\
\text { "Learn Young, Learn Fair", a stress } \\
\text { management program for fifth } \\
\text { and sixth graders: longitudinal } \\
\text { results from an experimental study }\end{array}$ & $\begin{array}{l}\text { Learn Young, } \\
\text { Learn Fair }\end{array}$ & Netherlands & $\begin{array}{l}\text { Cluster } \\
\text { Randomized } \\
\text { Controlled Trial }\end{array}$ & $\begin{array}{l}\text { Interv: } 693 \\
\text { students ( } 26 \\
\text { schools) } \\
\text { Control: } 732 \\
\text { students ( } 24 \\
\text { schools) }\end{array}$ & $\begin{array}{l}\text { - Improve stress management } \\
\text { and coping skills } \\
\text { - Reduce anxiety and depression } \\
\text { symptoms and incidence }\end{array}$ & $100 \%$ \\
\hline $\begin{array}{l}\text { Mishara (2006)[32] } \\
\text { Effectiveness of a mental health } \\
\text { promotion program to improve } \\
\text { coping skills in young children: } \\
\text { Zippy's Friends }\end{array}$ & $\begin{array}{l}\text { Zippy's } \\
\text { Friends }\end{array}$ & $\begin{array}{l}\text { Denmark \& } \\
\text { Lithuania }\end{array}$ & $\begin{array}{l}\text { Non-randomized } \\
\text { Experimental } \\
\text { Trial }\end{array}$ & $\begin{array}{l}\text { Students } \\
\text { Lithuania: } \\
\text { Interv: } 314 \\
\text { Control: } 104 \\
\text { Denmark: } \\
\text { Interv: } 322 \\
\text { Control: } 110\end{array}$ & $\begin{array}{l}\text { - Increase ability to cope with } \\
\text { everyday life adversities and } \\
\text { negative events } \\
\text { - Decrease problems that arise } \\
\text { from stressful situations } \\
\text { - Development of adaptive } \\
\text { coping skills }\end{array}$ & $75 \%$ \\
\hline $\begin{array}{l}\text { Clarke (2014)[33] } \\
\text { Evaluating the implementation } \\
\text { of a school-based emotional } \\
\text { well-being program: a cluster } \\
\text { randomized controlled trial of } \\
\text { Zippy's Friends for children } \\
\text { in disadvantaged primary schools }\end{array}$ & $\begin{array}{l}\text { Zippy's } \\
\text { Friends }\end{array}$ & Ireland & $\begin{array}{l}\text { Cluster } \\
\text { Randomized } \\
\text { Controlled Trial }\end{array}$ & $\begin{array}{l}\text { Interv: } 544 \\
\text { students } \\
\text { Control: } 222 \\
\text { students }\end{array}$ & $\begin{array}{l}\text { - Increase ability to cope with } \\
\text { everyday life adversities and } \\
\text { negative events } \\
\text { - Decrease problems that arise } \\
\text { from stressful situations } \\
\text { - Development of adaptive } \\
\text { coping skills }\end{array}$ & $25 \%$ \\
\hline $\begin{array}{l}\text { Dufour (2011)[34] } \\
\text { Improving Children's Adaptation: } \\
\text { New Evidence Regarding the Effectiveness } \\
\text { of Zippy's Friends, a School Mental Health } \\
\text { Promotion Program }\end{array}$ & $\begin{array}{l}\text { Zippy's } \\
\text { Friends }\end{array}$ & $\begin{array}{l}\text { Canada } \\
\text { (Quebec) }\end{array}$ & $\begin{array}{l}\text { Cluster } \\
\text { Randomized } \\
\text { Controlled Trial }\end{array}$ & $\begin{array}{l}\text { Interv: } 310 \\
\text { students (16 } \\
\text { classes) } \\
\text { Control: } 303 \\
\text { students (19 } \\
\text { classes) }\end{array}$ & $\begin{array}{l}\text { - Increase ability to cope with } \\
\text { everyday life adversities and } \\
\text { negative events } \\
\text { - Decrease problems that arise } \\
\text { from stressful situations } \\
\text { - Development of adaptive } \\
\text { coping skills }\end{array}$ & $50 \%$ \\
\hline $\begin{array}{l}\text { Holen (2012)[35] } \\
\text { The effectiveness of a universal school- } \\
\text { based program on coping and mental } \\
\text { health: a randomized, controlled study } \\
\text { of Zippy's Friends }\end{array}$ & $\begin{array}{l}\text { Zippy's } \\
\text { Friends }\end{array}$ & Norway & $\begin{array}{l}\text { Randomized } \\
\text { Controlled Trial }\end{array}$ & $\begin{array}{l}\text { Interv: } 686 \\
\text { students ( } 47 \\
\text { classes, } 18 \\
\text { schools) } \\
\text { Control: } 638 \\
\text { students ( } 44 \\
\text { classes, } 17 \\
\text { schools) }\end{array}$ & $\begin{array}{l}\text { - Increase ability to cope with } \\
\text { everyday life adversities and } \\
\text { negative events } \\
\text { - Decrease problems that arise } \\
\text { from stressful situations } \\
\text { - Development of adaptive } \\
\text { coping skills }\end{array}$ & $75 \%$ \\
\hline $\begin{array}{l}\text { Clarke (2015)[31] } \\
\text { Evaluating the implementation of an } \\
\text { emotional wellbeing program for } \\
\text { primary school children using } \\
\text { participatory methods }\end{array}$ & $\begin{array}{l}\text { Zippy's } \\
\text { Friends }\end{array}$ & Ireland & $\begin{array}{l}\text { Participatory } \\
\text { Workshop of } \\
\text { Randomized } \\
\text { Controlled Trial }\end{array}$ & $\begin{array}{l}\text { Interv: } 544 \\
\text { students } \\
\text { Control: } 222 \\
\text { students } \\
\text { Workshop: }\end{array}$ & $\begin{array}{l}\text { - Increase ability to cope with } \\
\text { everyday life adversities and } \\
\text { negative events } \\
\text { - Decrease problems that arise } \\
\text { from stressful situations } \\
\text { - Development of adaptive } \\
\text { coping skills }\end{array}$ & $100 \%$ \\
\hline $\begin{array}{l}\text { Nielsen (2015)[37] } \\
\text { Promotion of social and emotional } \\
\text { competence: Experiences from a } \\
\text { mental health intervention applying } \\
\text { a whole school approach }\end{array}$ & Up & Denmark & $\begin{array}{l}\text { Multi- } \\
\text { component } \\
\text { Intervention, No } \\
\text { Control Group }\end{array}$ & $\begin{array}{l}589 \text { students } \\
\text { (2 schools) }\end{array}$ & $\begin{array}{l}\text { - Enhance social and emotional } \\
\text { competencies to improve } \\
\text { mental health } \\
\text { - Increase positivity of school } \\
\text { mental health environment }\end{array}$ & $50 \%$ \\
\hline $\begin{array}{l}\text { Caldarella (2009)[40] } \\
\text { Promoting Social and Emotional Learning } \\
\text { in Second Grade Students: A Study of } \\
\text { the Strong Start Curriculum }\end{array}$ & Strong Start & $\begin{array}{l}\text { United } \\
\text { States } \\
\text { (Utah) }\end{array}$ & $\begin{array}{l}\text { Quasi- } \\
\text { Experimental, } \\
\text { Non-Equivalent } \\
\text { Control Group }\end{array}$ & 26 students & $\begin{array}{l}\text { - Prevent future emotional and } \\
\text { behavioral problems via the } \\
\text { promotion of social and } \\
\text { emotional wellbeing }\end{array}$ & $50 \%$ \\
\hline
\end{tabular}


Table 1 Summary of Articles Included in the Review (Continued)

\begin{tabular}{|c|c|c|c|c|c|c|}
\hline First author, year published Study type & $\begin{array}{l}\text { Program } \\
\text { Name }\end{array}$ & Location & Study Type & Sample Size & Aim of Program and Study & $\begin{array}{l}\text { MMAT } \\
\text { Score }\end{array}$ \\
\hline $\begin{array}{l}\text { Yamamoto (2017) [38] } \\
\text { Effects of the cognitive behavioral You } \\
\text { Can Do It! Education program on the } \\
\text { resilience of Japanese elementary school } \\
\text { students: A preliminary investigation }\end{array}$ & $\begin{array}{l}\text { You Can Do } \\
\text { It! Education }\end{array}$ & Tokyo & $\begin{array}{l}\text { Quasi- } \\
\text { Experimental, } \\
\text { Intervention, } \\
\text { Control Group }\end{array}$ & $\begin{array}{l}125 \text { students, } \\
\text { intervention } \\
n=78, \text { control } \\
\text { group }=47\end{array}$ & $\begin{array}{l}\text { - Evaluate a mental health } \\
\text { promotion program's efficacy } \\
\text { in enhancing resilience in } \\
\text { schools }\end{array}$ & $100 \%$ \\
\hline
\end{tabular}

Do It! Education program in Japan, where program staff translated and altered the internationally-implemented program with Japan-specific illustrations, examples and exercises to optimize the connection with students [38]. Three studies identified problems with implementation of programming due to teacher perceptions, time constraints, participation rates and class literacy levels $[32,33,38,40]$.

\section{Evaluation frameworks, tools and indicators}

Study evaluation frameworks and indicators (summarised in Table 3) are reported with more detail on evaluation tools and methods used for evaluating elements of programing reported in Appendix. Studies varied greatly on the timing and purpose of their evaluation although all applied a combination of pre-assessment, post-assessment, process evaluation, implementation evaluation and follow up assessments. Within specific programs, different evaluations were used for different implementations and contexts. The five articles reporting on the Zippy's Friends program utilized different evaluation methods [31-35]. Mishara and Ystgaard (2006) evaluated the implementation of Zippy's Friends in two countries with similar socio-demographic characteristics, Lithuania and Denmark, and found similar results in outcomes of students in the intervention groups in both countries. Yamamoto et al. used a semi-experimental design with intervention and control groups and utilized three self-report scales to evaluate students [38]. Clarke evaluated a randomized-controlled trial implementation of Zippy's Friends in Ireland using both standard measures [33] and a participatory workshop with a subsample of students. The workshop was semi-structured around three key themes: lived experiences and coping reactions; emotion recognition and regulation; and program evaluation [31]. In all articles meeting out inclusion criteria, multiple standardized, validated tools were used for evaluation measures, most commonly the Children's Depression Inventory (CDI, Short or Complete Form) [30, 39], the Strengths and Difficulties Questionnaire [33, 35], the Schoolagers' Coping Strategies Inventory [32, 34], and a Program Fidelity Checklist $[33,40]$. Evaluation methods commonly included in-class observations [33, 34, 36, 40], researcher-developed questionnaires $[34,36]$ and session reports [32, 34, 35].

\section{Outcomes}

Each article identified outcomes associated with their research question and hypothesis with outcomes following program implementation to assess the impact of the program. Table 4 presents a summary of whether major outcomes were considered by the article to have changed as a result of programing. In eight studies, researchers identified at baseline an overarching need for resilience programing among students, including low levels of trust and empathy; problems with emotion control, relationships and help-seeking; or reported symptoms [30, 31, 33, 36-40]. Ten out of eleven studies reported positive outcomes with improvements in student resilience and protective factors, including frequency of use of coping skills, internalizing behaviors, and self-efficacy at post-assessment [30-34, 36-40]. Three studies identified shortcomings in outcomes despite positive results from the overall program implementation and outcomes. Kraag et al. (2009) identified a lack of follow up and social reinforcement for components taught in programing, with negative implications on long-term follow-up outcomes [39]. Clarke and colleagues (2014) showed limited effects on resilience itself, but highlighted a marked increase in self-awareness among students [33]. Variations in outcomes between informants was highlighted in Holen et al (2012) who did not determine that resilience itself was an outcome of the program [35].

\section{Discussion}

This review examined the program criteria and outcome measures used in the implementation and evaluation of resilience-focused, universal, school-based mental health promotion programs. Eleven published studies based on seven different programs were identified and included.

\section{Characteristics of effective programs}

Several characteristics of effective programing stood out. The involvement of teachers in the delivery of programs 


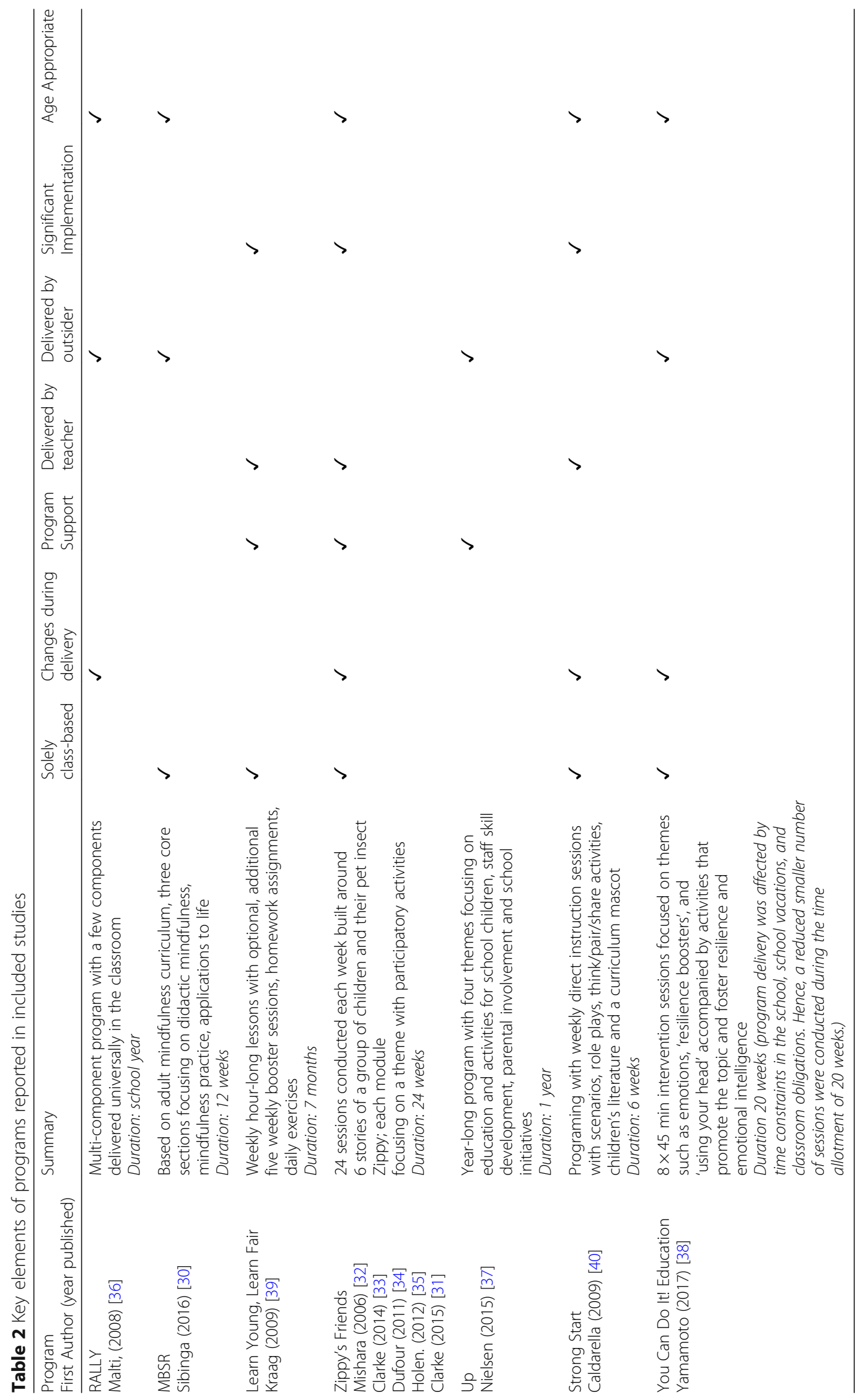


Table 3 Evaluation frameworks of included studies

\begin{tabular}{|c|c|c|c|c|c|c|c|}
\hline $\begin{array}{l}\text { First author } \\
\text { (Year published) } \\
\text { Study }\end{array}$ & Evaluator & Indicators & $\begin{array}{l}\text { Pre- } \\
\text { Asses. }\end{array}$ & $\begin{array}{l}\text { Process/ } \\
\text { Implmt. }\end{array}$ & $\begin{array}{l}\text { Post- } \\
\text { Asses. }\end{array}$ & $\begin{array}{l}\text { Follow } \\
\text { Up }\end{array}$ & Tools (See Appendix) \\
\hline $\begin{array}{l}\text { Malti. (2008) [36] } \\
\text { RALLY }\end{array}$ & Study Researchers & $\begin{array}{l}\text { Development, resilience } \\
\text { techniques, symptoms, } \\
\text { relationships } \\
\text { Program implementation }\end{array}$ & $\checkmark$ & $\checkmark$ & $\checkmark$ & & $\begin{array}{l}\text { SRM-SF; Researcher-developed } \\
\text { resilience scale; YSR }\end{array}$ \\
\hline $\begin{array}{l}\text { Sibinga (2016) } \\
\text { [30] } \\
\text { MBSR }\end{array}$ & Program Staff & $\begin{array}{l}\text { Mindfulness, psychological } \\
\text { symptoms, anxiety, mood } \\
\text { and emotion regulation, } \\
\text { coping }\end{array}$ & $\checkmark$ & & $\checkmark$ & & $\begin{array}{l}\text { CDI-S; SCL-90-R; MASC; PANAS; } \\
\text { DES; STAXI-2; CRSQ; CSE }\end{array}$ \\
\hline $\begin{array}{l}\text { Kraag (2009) } \\
\text { [39] } \\
\text { Learn Young, } \\
\text { Learn Fair }\end{array}$ & $\begin{array}{l}\text { Maastricht University } \\
\text { students }\end{array}$ & $\begin{array}{l}\text { Stress management, coping, } \\
\text { anxiety, depression }\end{array}$ & $\checkmark$ & & $\checkmark$ & $\checkmark$ & STAIC; DIC-SF; MUSIC; SPSI \\
\hline $\begin{array}{l}\text { Mishara (2006) } \\
{[32]} \\
\text { Zippy's Friends }\end{array}$ & Independent researchers & $\begin{array}{l}\text { Student engagement, mood, } \\
\text { behavior and emotion } \\
\text { regulation, coping skills } \\
\text { Program implementation }\end{array}$ & $\checkmark$ & $\checkmark$ & $\checkmark$ & & $\begin{array}{l}\text { Session reports; interviews; } \\
\text { Social Skills Questionnaire; } \\
\text { SSQTF; Schoolagers Coping } \\
\text { Strategies Inventory; SSQSF }\end{array}$ \\
\hline $\begin{array}{l}\text { Clarke (2014) } \\
\text { [33] } \\
\text { Zippy's Friends }\end{array}$ & $\begin{array}{l}\text { Researcher \& Health } \\
\text { Promotion Specialist }\end{array}$ & $\begin{array}{l}\text { Social and emotional literacy, } \\
\text { social and emotional behavior } \\
\text { Program implementation }\end{array}$ & $\checkmark$ & $\checkmark$ & $\checkmark$ & $\checkmark$ & $\begin{array}{l}\text { Emotional Literacy Checklist; } \\
\text { SDQ; Program Fidelity Checklist }\end{array}$ \\
\hline $\begin{array}{l}\text { Dufour (2011) } \\
\text { [34] } \\
\text { Zippy's Friends }\end{array}$ & $\begin{array}{l}\text { Undergraduate university } \\
\text { students }\end{array}$ & $\begin{array}{l}\text { Coping mechanisms, } \\
\text { socio-emotional functioning, } \\
\text { perceived social support, } \\
\text { classroom climate } \\
\text { Program implementation }\end{array}$ & $\checkmark$ & $\checkmark$ & $\checkmark$ & & $\begin{array}{l}\text { Observations; Session reports; } \\
\text { Schoolagers Coping Strategy } \\
\text { Inventory; Surveys; Socio-Emotional } \\
\text { Profile; Social Support Scale for } \\
\text { Children; Class Environment Climate } \\
\text { Questionnaire }\end{array}$ \\
\hline $\begin{array}{l}\text { Holen (2012) } \\
\text { [35] } \\
\text { Zippy's Friends }\end{array}$ & $\begin{array}{l}\text { Teachers \& Study } \\
\text { Researcher }\end{array}$ & Coping skills & $\checkmark$ & & $\checkmark$ & & KidCope Questionnaire; SDQ \\
\hline $\begin{array}{l}\text { Clarke (2015) } \\
\text { [31] } \\
\text { Zippy's Friends }\end{array}$ & Study Researcher & $\begin{array}{l}\text { Coping skills, emotional } \\
\text { literacy } \\
\text { Program implementation }\end{array}$ & $\checkmark$ & $\checkmark$ & $\checkmark$ & & $\begin{array}{l}\text { Participatory workshop; draw and } \\
\text { write technique; vignette response } \\
\text { feelings activity; brainstorming }\end{array}$ \\
\hline $\begin{array}{l}\text { Nielsen (2015) } \\
\text { [37] } \\
\text { Up }\end{array}$ & $\begin{array}{l}\text { Child and Adolescent } \\
\text { Health Research Group at } \\
\text { NIPH }\end{array}$ & $\begin{array}{l}\text { Assertiveness, empathy, } \\
\text { collaborative skills }\end{array}$ & $\checkmark$ & $\checkmark$ & $\checkmark$ & $\checkmark$ & Anonymous Surveys \\
\hline $\begin{array}{l}\text { Caldarella (2009) } \\
{[40]} \\
\text { Strong Start }\end{array}$ & $\begin{array}{l}\text { Teachers \& Research } \\
\text { Assistants }\end{array}$ & $\begin{array}{l}\text { Internalizing and externalizing } \\
\text { behaviors, peer-related } \\
\text { pro-social behavior } \\
\text { Program implementation }\end{array}$ & $\checkmark$ & $\checkmark$ & $\checkmark$ & & $\begin{array}{l}\text { SSRS; Observations; Program fidelity } \\
\text { checklist; IRP-15; Student } \\
\text { Self-Assessment of Social Validity }\end{array}$ \\
\hline $\begin{array}{l}\text { Yamamoto } \\
\text { (2017) [38] } \\
\text { You Can Do It! } \\
\text { Education }\end{array}$ & Study Researchers & $\begin{array}{l}\text { Anxiety, Awareness of Social } \\
\text { Support, Resilience }\end{array}$ & $\checkmark$ & & $\checkmark$ & & $\begin{array}{l}\text { Spence children's anxiety scale (SCAS), } \\
\text { Social support scale for children (SSSC), } \\
\text { Resilience in elementary school } \\
\text { children scale (RESC) }\end{array}$ \\
\hline
\end{tabular}

assess assessment, implmt implementation

emerged as key. Numerous studies used teachers to deliver the program, a feature presented positively as providing the opportunity for adaptability of programing and more seamless implementation, if provided with programmatic support and training. For example, the Zippy's Friends program uses teachers to deliver the content materials [33] and teachers reported receiving substantial, helpful program support by research and program staff.

In their review of factors of success for implementation, adaptation of programing was identified as a key component of implementation [38, 41]. Teachers of the
Zippy's Friends Program reported the ability to adapt, add and remove activities relating to thematic content based on student literacy, mood and timing, as one of the most important parts of program delivery [33]. This allowed the maintenance of high program fidelity while also involving students in the most effective way possible. Teachers are an important resource in the development of children's resilience, as they already have rapport and an understanding of the students and are more likely to know their students lived experiences and current coping and help-seeking strategies. Yamamoto et al. credit their successful implementation of the 


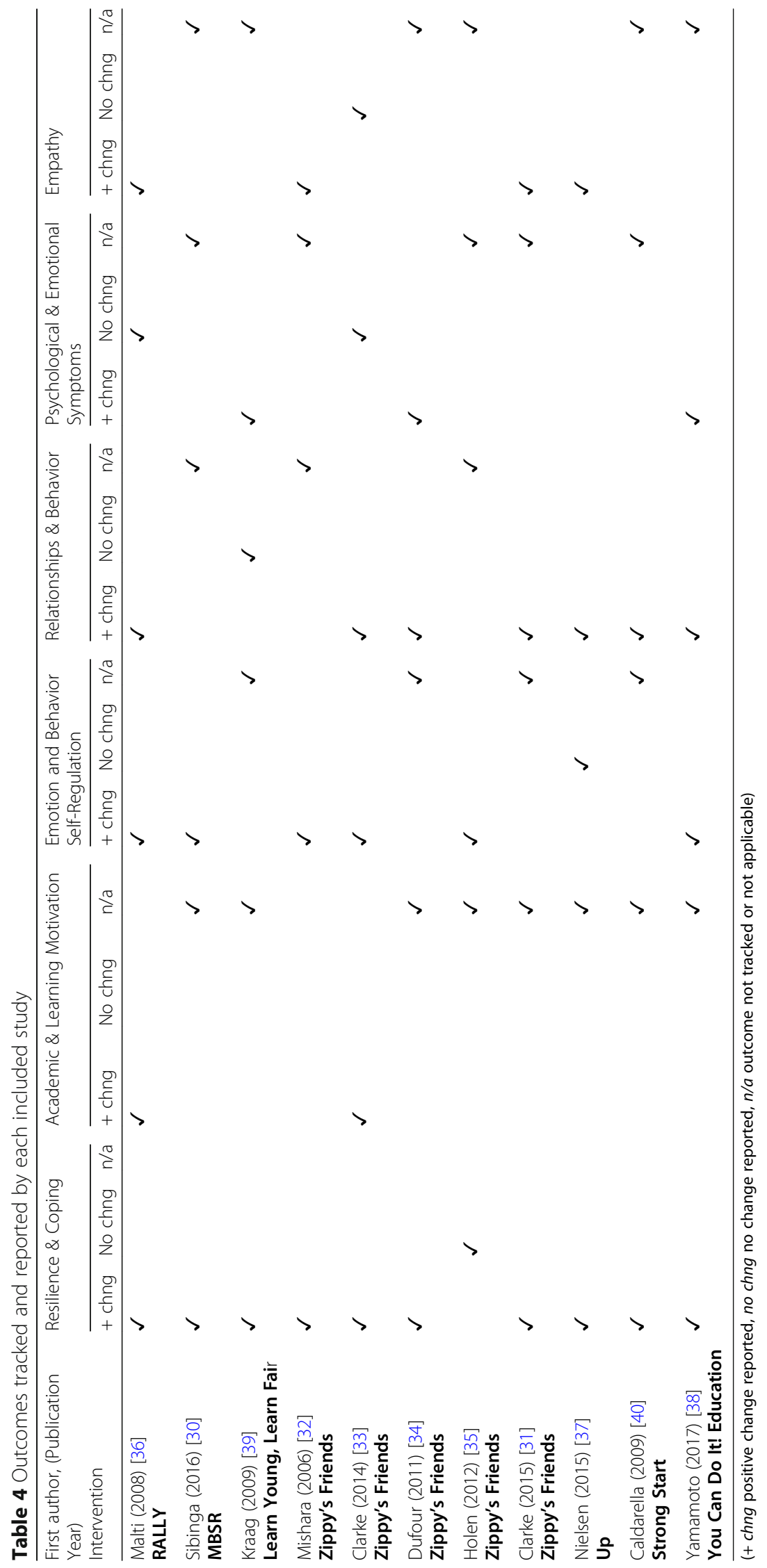


YCDI! Program with the extensive edits to the curriculum to adapt it to Japanese culture and relationships [38].

The length of programing did not appear to impact on the number of outcomes achievable. The RALLY program ran for an entire school year and provided consistent resilience outcomes [36], while the Mindfulness-Based Stress Reduction program ran for only 12 weeks and showed positive resilience outcomes as well [30]. The YCDI! Program ran for a shorter period of time than most implementations of the program but still demonstrated significant results [38]. Importance was placed on the intensity of sessions and the content delivered, as opposed to the regularity. However, importantly, if follow up evaluations were conducted, they did not reveal that outcomes were maintained in the longer term after most programs. This suggests that program length may not alter the ongoing resiliency of students once the program ends.

\section{Emergent themes across studies}

Although all eleven articles presented programs that aimed at fostering the resilience skills and protective factors of students, the specific skills and outcomes taught in each program differed. This is consistent with research highlighting the difficulty that exists in defining resilience and creating programs around the topic [1]. Not only is the definition difficult and variable between studies, but the criteria and skills that come with developing resilience differ as well. In the RALLY study, researchers targeted resilience, and the outcomes evaluated were empathy, trust of others, and emotional regulation skills [36]. On the other hand, the UP study targeted resilience through social and emotional competencies that allow students to engage and navigate daily life, social interactions and society [37]. Both programs aimed to foster social and emotional development by increasing resilience skills and protective factors, but were based on differences in terminology and theory. Evaluations of both programs determined they had a positive outcome on resilience in students despite these differences.

An effect noted by a number of studies included in this review was the "ceiling effect" since many of the students enrolled in universal-based programs have high baseline mental health and social and emotional competence [33]. Although individuals within the group might suffer from higher risk factors or mental illness, across the board students present with generally normal levels. As such, when the program is implemented, outcomes may be generated but will not be large as there is little room for change. This is not the case when providing targeted programs with students who all generally have much more room for change, given that they begin the program with lower scores at baseline. Despite the ceiling effect, research has shown that resilience-boosting programing benefits at-risk but are not specific for at-risk children. Additionally, properly identifying and screening target groups for targeted programing is often unsuccessful due to the complexities of mental health, and preventive approaches, such as universal resilience-boosting programing, are considered the most all-encompassing method [42]. As such, a program promoting resilience will support positive changes and growth in both groups of kids, although with more significant differences in the at-risk group.

\section{Characteristics and methodologies of evaluations}

An element of the evaluations that emerged in many articles is the removed nature of evaluation when collecting data on children's capacities. Many of the programs seek to foster resilience through the development of coping skills, and use scales or observations in order to measure outcomes. The Learn Young, Learn Fair program evaluated a positive effect on emotion-focused, adaptive coping skills using validated questionnaires and scales [39]. This approach is used in all the program evaluations, but does not leave room for lived experiences to be factored into the interpretation of outcomes. These traditional evaluation methodologies can be seen as researching on a topic, rather than researching for a cause or population, as they do not leave room for ambiguity or other factors.

Additionally, a couple of studies in this review used evaluation tools that did not take into account the views of children themselves. The researchers chose to interview and evaluate both teachers' and the program deliverers' perceptions and ratings, rather than interviewing or evaluating the children themselves. For example, Caldarella, Christensen et al. (2009) evaluate children's outcomes through pre- and post-assessments of the teacher's perceptions of her students, using validated assessment tools [40]. However, evaluations like this introduce an additional limitation to the outcome analysis, as they gather data through secondary sources with the program delivered to children for their benefit, but outcomes not gathered directly from the children. However, observational data is a key component of a program evaluation with many studies successfully using observations to ensure program fidelity and as part of process evaluations.

More insight around outcomes occurs when multiple evaluation tools and methods are used [43]. Clarke and colleagues (2015) evaluated the use of a participatory workshop determining children's coping skills which used draw and write techniques that allowed children to 
share their feelings using their own words rather than those of researchers [44], as well as vignettes to eliminate interview processes [45]. Students from the intervention group were found to use more adaptive coping skills in their daily life, both in and out of the classroom than children in the control group [31]. These results were supported by the quantitative data collected on the larger student sample from which the participatory workshop subsample was drawn [33]. A clearer picture of children's coping skills and experiences with the Zippy's Friends program was gathered through the use of both qualitative and quantitative evaluation methodology. Additionally, children's lived experiences and direct insights were gathered through the participatory workshop model, allowing for a greater breadth of understanding on the program's efficiency.

\section{Limitations of articles and evidence}

Consideration must be given to the ethics and feasibility of implementing and evaluating programs for mental health promotion. Ethical concerns arise from providing a program that might be highly beneficial for a group of children, and not for another, essentially disadvantaging them. The ethics are further confounded by the lack of complete or stringent randomization described in the studies that include a control group. To avoid the dilemma of disadvantaging students, studies on success factors have highlighted that in many studies the control groups do not receive 'no intervention' [41]. For example, Sibinga et al. (2016) included an active control group. While the intervention group received the Mindfulness-Based Stress Reduction program being studied, the control group received Healthy Topics, a general health program to match the MBSR structure. Thus, while the control group students are not receiving a resilience-focused, mental health promotion program, they still receive a health promotion program but one which allows a distinction between control and intervention groups around resilience outcomes and mental health [30]. Yamamoto and colleagues, however, did not provide programing to the control group following the intervention [38].

The evidence provided by certain articles must be weighed with differing criteria. Seven articles evaluated a program against a control group, allowing for comparison of outcomes. These articles present more substantial outcome evidence than those that do not include a control group for comparison. For example, Nielsen and colleagues (2015) and Caldarella and colleagues (2009) did not have a control group, decreasing the strength of their evaluation. Nielsen et al. (2015) implemented the UP program in kindergarten through grade 9, but only evaluated grades 5-9. Such selective evaluation introduces potential bias and paired with the absence of a control group makes it difficult to identify if the increase in social and emotional competencies is due to the UP intervention, or simply a natural developmental progression [37].

A limitation of the evaluations in many programs is the involvement of the person delivering the program as the evaluator. This can be seen in many studies on the Zippy's Friends program, where the classroom teacher delivers the program and conducts the process and implementation evaluation themselves. Third-party observations are sometimes conducted in addition to verify program fidelity and implementation outcomes. Of note is that observational evaluation and the use of independent evaluators have been more extensively documented as reliable than using tools based on self-report [41].

We also note that despite gender differences in the prevalence of mental health problems and the type of resilience protective factors that children and adolescents use, the studies did not generally report results by gender [46, 47]. This limitation could be overcome by encouraging that future studies provide a gender breakdown or highlight gender-specific results.

\section{Conclusion}

This review complements previous reviews on mental health promotion programing for students. Our focus on universally delivered programs in primary schools reveals key components and strengths of programing that make for the most successful delivery and evaluation and enables important conclusions to be drawn.

The review confirms that adaptability and teacher involvement are key elements of program delivery, with student engagement and use of multiple methods strengthening program evaluation. The use of participatory methods to engage children allows for greater assessment of lived experiences and use of coping skills compared to self-reporting tools or observations. Adaptability of curriculum to different contexts, seen in the Zippy's Friends program, was considered successful by multiple authors, illustrating that broad program application across multiple contexts is possible and effective.

This review demonstrates the importance of establishing key criteria to be measured during delivery and evaluation of youth mental health promotion programs, particularly in terms of defining resilience and its associated indicators. The successes of the programs detailed by the studies included in this review highlight the need for and benefits of such programs. Further research on primary-school, universally delivered mental health promotion programs could be conducted in specific contexts, particularly more difficult settings such as developing countries or conflict zones. 


\section{Appendix}

Table $\mathbf{5}$ Evaluation tools and methodologies used in included studies

\begin{tabular}{|c|c|c|c|c|c|}
\hline Criteria & Tool & $\begin{array}{l}\text { First author } \\
\text { (Year) of } \\
\text { Studies in } \\
\text { which Tool } \\
\text { was Used }\end{array}$ & Purpose & Methodology & Timeframe \\
\hline Depression & $\begin{array}{l}\text { Children's } \\
\text { Depression } \\
\text { Inventory, Short } \\
\text { or Complete } \\
\text { Form } \\
\text { CDI }\end{array}$ & $\begin{array}{l}\text { Sibinga } \\
(2016)[30] \\
\text { Kraag } \\
(2009)[39]\end{array}$ & $\begin{array}{l}\text { Assess depressive } \\
\text { symptoms }\end{array}$ & Self-reported survey & $\begin{array}{l}\text { Pre- assessment } \\
\text { Post-assessment } \\
\text { Follow up }\end{array}$ \\
\hline \multirow[t]{3}{*}{ Anxiety } & $\begin{array}{l}\text { Multidimensional } \\
\text { Anxiety Scale for } \\
\text { Children } \\
\text { MASC }\end{array}$ & $\begin{array}{l}\text { Sibinga } \\
\text { (2016) [30] }\end{array}$ & Assess anxiety symptoms & Self-reported survey & $\begin{array}{l}\text { Pre-assessment } \\
\text { Post-assessment }\end{array}$ \\
\hline & $\begin{array}{l}\text { Spence Children's } \\
\text { Anxiety Scale } \\
\text { SCAS }\end{array}$ & $\begin{array}{l}\text { Yamamoto } \\
\text { (2017) [38] }\end{array}$ & $\begin{array}{l}\text { Assess frequency of anxiety } \\
\text { symptoms }\end{array}$ & Self-reported survey & $\begin{array}{l}\text { Pre-assessment } \\
\text { Post-assessment }\end{array}$ \\
\hline & $\begin{array}{l}\text { Spielberger's } \\
\text { State-Trait Anx- } \\
\text { iety Inventory for } \\
\text { Children } \\
\text { STAIC }\end{array}$ & $\begin{array}{l}\text { Kraag } \\
\text { (2009) [39] }\end{array}$ & Assess anxiety symptoms & Self-reported survey & $\begin{array}{l}\text { Pre-assessment } \\
\text { Post-assessment } \\
\text { Follow up }\end{array}$ \\
\hline $\begin{array}{l}\text { Social/ } \\
\text { Cognitive } \\
\text { Development }\end{array}$ & $\begin{array}{l}\text { Socio-moral } \\
\text { Reflection } \\
\text { Measure, Short } \\
\text { Form } \\
\text { SRM-SF }\end{array}$ & $\begin{array}{l}\text { Malti (2008) } \\
{[36]}\end{array}$ & $\begin{array}{l}\text { Assess developmental levels } \\
\text { of cognitive-moral skills }\end{array}$ & $\begin{array}{l}\text { Paper and pencil self-reported } \\
\text { survey }\end{array}$ & $\begin{array}{l}\text { Pre-assessment } \\
\text { Post-assessment }\end{array}$ \\
\hline \multirow[t]{3}{*}{ Resilience } & $\begin{array}{l}\text { Researcher- } \\
\text { developed } \\
\text { Resilience Scale }\end{array}$ & $\begin{array}{l}\text { Malti (2008) } \\
{[36]}\end{array}$ & $\begin{array}{l}\text { Measure selected basic } \\
\text { resilience factors, emotional } \\
\text { regulation skills, academic skills }\end{array}$ & Self-reported survey & $\begin{array}{l}\text { Pre-assessment } \\
\text { Post-Assessment }\end{array}$ \\
\hline & $\begin{array}{l}\text { Resilience in } \\
\text { Elementary } \\
\text { School Children } \\
\text { Scale } \\
\text { RESC }\end{array}$ & $\begin{array}{l}\text { Yamamoto } \\
(2017)[38]\end{array}$ & $\begin{array}{l}\text { Measure } 19 \text { elements relating } \\
\text { to aspects of resilience }\end{array}$ & Self-reported & $\begin{array}{l}\text { Pre-assessment } \\
\text { Post-assessment }\end{array}$ \\
\hline & $\begin{array}{l}\text { Socio-Emotional } \\
\text { Profile } \\
\text { (Dumas et al, } \\
\text { 1997) }\end{array}$ & $\begin{array}{l}\text { Dufour } \\
\text { (2011) [34] }\end{array}$ & $\begin{array}{l}\text { Measure social competencies } \\
\text { and adaption problems }\end{array}$ & $\begin{array}{l}80 \text { items on six point scale, } \\
\text { self-reported survey }\end{array}$ & $\begin{array}{l}\text { Pre-assessment } \\
\text { Post-assessment }\end{array}$ \\
\hline Mindfulness & $\begin{array}{l}\text { Children's } \\
\text { Acceptance and } \\
\text { Mindfulness } \\
\text { Measure }\end{array}$ & $\begin{array}{l}\text { Sibinga } \\
\text { (2016) [30] }\end{array}$ & Measure of mindfulness & 10 item, self-reported survey & $\begin{array}{l}\text { Pre-assessment } \\
\text { Post-assessment }\end{array}$ \\
\hline \multirow[t]{2}{*}{ Symptoms } & $\begin{array}{l}\text { Youth Self Report } \\
\text { YSR }\end{array}$ & $\begin{array}{l}\text { Malti (2008) } \\
{[36]}\end{array}$ & $\begin{array}{l}\text { Assess behavioral and } \\
\text { emotional functioning and } \\
\text { symptoms }\end{array}$ & Self-reported survey & $\begin{array}{l}\text { Pre-assessment } \\
\text { Post-assessment }\end{array}$ \\
\hline & $\begin{array}{l}\text { Symptoms } \\
\text { Checklist 90-R } \\
\text { SCL-90-R }\end{array}$ & $\begin{array}{l}\text { Sibinga } \\
\text { (2016) [30] }\end{array}$ & $\begin{array}{l}\text { Assess paranoid ideation, } \\
\text { hostility, somatization }\end{array}$ & Self-reported survey & $\begin{array}{l}\text { Pre-assessment } \\
\text { Post-assessment }\end{array}$ \\
\hline \multirow[t]{2}{*}{ Stress } & $\begin{array}{l}\text { Children's Post- } \\
\text { Traumatic Symp- } \\
\text { tom Severity } \\
\text { Checklist } \\
\text { CPSS }\end{array}$ & $\begin{array}{l}\text { Sibinga } \\
\text { (2016) [30] }\end{array}$ & $\begin{array}{l}\text { Assess stress symptom } \\
\text { severity and frequency }\end{array}$ & Self-reported survey & $\begin{array}{l}\text { Pre-assessment } \\
\text { Post-assessment }\end{array}$ \\
\hline & $\begin{array}{l}\text { Maastricht } \\
\text { University Stress } \\
\text { Instrument for }\end{array}$ & $\begin{array}{l}\text { Kraag } \\
\text { (2009) [39] }\end{array}$ & $\begin{array}{l}\text { Assess stress symptom } \\
\text { severity and frequency }\end{array}$ & $\begin{array}{l}\text { Self-reported scale survey } \\
\text { Developed for study }\end{array}$ & $\begin{array}{l}\text { Pre-assessment } \\
\text { Post-assessment } \\
\text { Followup }\end{array}$ \\
\hline
\end{tabular}


Table 5 Evaluation tools and methodologies used in included studies (Continued)

\begin{tabular}{|c|c|c|c|c|c|}
\hline Criteria & Tool & $\begin{array}{l}\text { First author } \\
\text { (Year) of } \\
\text { Studies in } \\
\text { which Tool } \\
\text { was Used }\end{array}$ & Purpose & Methodology & Timeframe \\
\hline \multirow[t]{4}{*}{ Relationships } & $\begin{array}{l}\text { Social Support } \\
\text { Scale for Children }\end{array}$ & $\begin{array}{l}\text { Dufour } \\
\text { (2011) [34] } \\
\text { Yamamoto } \\
\text { (2017) [38] }\end{array}$ & $\begin{array}{l}\text { Measure perceived } \\
\text { social support by } \\
\text { children: social support } \\
\text { from parents, teachers, } \\
\text { peers in class } \\
\text { and intimate friends }\end{array}$ & 24 items on four point scale & $\begin{array}{l}\text { Pre-assessment } \\
\text { Post-assessment }\end{array}$ \\
\hline & $\begin{array}{l}\text { School Social } \\
\text { Behavior Skills } \\
\text { SSBS }\end{array}$ & $\begin{array}{l}\text { Caldarella } \\
\text { (2009) [40] }\end{array}$ & $\begin{array}{l}\text { Evaluate social competence } \\
\text { and antisocial behavior of } \\
\text { children }\end{array}$ & $\begin{array}{l}\text { Norm-referenced, } \\
\text { self-reported survey }\end{array}$ & $\begin{array}{l}\text { Pre-assessment } \\
\text { Post-assessment }\end{array}$ \\
\hline & $\begin{array}{l}\text { Social Skills } \\
\text { Rating System } \\
\text { SSRS }\end{array}$ & $\begin{array}{l}\text { Caldarella } \\
\text { (2009) [40] }\end{array}$ & $\begin{array}{l}\text { Evaluate pro-social skills } \\
\text { and problem behaviors } \\
\text { of students }\end{array}$ & $\begin{array}{l}\text { Six-subscale items, } \\
\text { norm-referenced, } \\
\text { self-reported survey }\end{array}$ & $\begin{array}{l}\text { Pre-assessment } \\
\text { Post-assessment }\end{array}$ \\
\hline & $\begin{array}{l}\text { Social Skills } \\
\text { Questionnaire } \\
\text { Teacher Form, } \\
\text { Elementary Level } \\
\text { SSQTF } \\
\text { Student Form, } \\
\text { Elementary Level } \\
\text { SSQSF }\end{array}$ & $\begin{array}{l}\text { Mishara } \\
(2006)[32]\end{array}$ & $\begin{array}{l}\text { Measure frequency of } \\
\text { observed behaviors and } \\
\text { social skills; } \\
\text { scores determined for } \\
\text { cooperation, assertion, } \\
\text { self-control }\end{array}$ & $\begin{array}{l}\text { Rating of frequency of } \\
\text { specific behaviors }\end{array}$ & $\begin{array}{l}\text { Process } \\
\text { evaluation }\end{array}$ \\
\hline \multirow[t]{8}{*}{$\begin{array}{l}\text { Mood \& } \\
\text { Emotions }\end{array}$} & $\begin{array}{l}\text { Positive and } \\
\text { Negative Affect } \\
\text { Schedule } \\
\text { PANAS }\end{array}$ & $\begin{array}{l}\text { Sibinga } \\
(2016)[30]\end{array}$ & $\begin{array}{l}\text { Assess mood and emotion } \\
\text { regulation }\end{array}$ & Self-reported survey & $\begin{array}{l}\text { Pre-assessment } \\
\text { Post-assessment }\end{array}$ \\
\hline & $\begin{array}{l}\text { Differential } \\
\text { Emotional Scale } \\
\text { DES }\end{array}$ & $\begin{array}{l}\text { Sibinga } \\
(2016)[30]\end{array}$ & $\begin{array}{l}\text { Assess mood and emotion } \\
\text { regulation }\end{array}$ & Self-reported survey & $\begin{array}{l}\text { Pre-assessment } \\
\text { Post-assessment }\end{array}$ \\
\hline & Aggression Scale & $\begin{array}{l}\text { Sibinga } \\
(2016)[30]\end{array}$ & $\begin{array}{l}\text { Assess mood and emotion } \\
\text { regulation }\end{array}$ & Self-reported survey & $\begin{array}{l}\text { Pre-assessment } \\
\text { Post-assessment }\end{array}$ \\
\hline & $\begin{array}{l}\text { State-Trait Anger } \\
\text { Expression } \\
\text { Inventory } \\
\text { STAXI-2 }\end{array}$ & $\begin{array}{l}\text { Sibinga } \\
(2016)[30]\end{array}$ & $\begin{array}{l}\text { Assess mood and emotion } \\
\text { regulation }\end{array}$ & Self-reported survey & $\begin{array}{l}\text { Pre-assessment } \\
\text { Post-assessment }\end{array}$ \\
\hline & $\begin{array}{l}\text { Strengths and } \\
\text { Difficulties } \\
\text { Questionnaires } \\
\text { SDQ }\end{array}$ & $\begin{array}{l}\text { Clarke } \\
\text { (2014) [33] } \\
\text { Holen } \\
\text { (2012) [35] }\end{array}$ & $\begin{array}{l}\text { Assess children's emotional } \\
\text { and behavioral functioning: } \\
\text { emotional symptoms, conduct } \\
\text { 2problems, hyperactivity, peer } \\
\text { relationship problems, } \\
\text { pro-social behavior }\end{array}$ & $\begin{array}{l}\text { Self-reported questionnaire, } \\
25 \text { items with five main } \\
\text { subscale scores }\end{array}$ & $\begin{array}{l}\text { Pre-assessment } \\
\text { Post-assessment } \\
\text { Follow up }\end{array}$ \\
\hline & Feelings Activity & $\begin{array}{l}\text { Clarke } \\
\text { (2015) [31] }\end{array}$ & $\begin{array}{l}\text { Assess ability to identify } \\
\text { feelings in response to } \\
\text { problem situations }\end{array}$ & $\begin{array}{l}\text { Part of participatory workshop } \\
\text { evaluation: } 6 \text { vignettes read } \\
\text { aloud and children asked to } \\
\text { respond and explain each }\end{array}$ & $\begin{array}{l}\text { Process } \\
\text { evaluation } \\
\text { Post-assessment }\end{array}$ \\
\hline & $\begin{array}{l}\text { Social and } \\
\text { Emotional } \\
\text { Competence } \\
\text { Index }\end{array}$ & $\begin{array}{l}\text { Nielsen } \\
\text { (2015) [37] }\end{array}$ & $\begin{array}{l}\text { Assess assertiveness, } \\
\text { empathy and collaborative } \\
\text { skills }\end{array}$ & $\begin{array}{l}\text { Self-reported questionnaire } \\
\text { with ranked answers }\end{array}$ & $\begin{array}{l}\text { Pre-assessment } \\
\text { Post-assessment }\end{array}$ \\
\hline & $\begin{array}{l}\text { Emotional } \\
\text { Literacy Checklist }\end{array}$ & $\begin{array}{l}\text { Clarke } \\
\text { (2014) [33] }\end{array}$ & $\begin{array}{l}\text { Assess emotional literacy: } \\
\text { self-awareness, self-regulation, } \\
\text { motivation, empathy and } \\
\text { social skills }\end{array}$ & $\begin{array}{l}\text { Self-reported questionnaire, } 20 \text { items } \\
\text { rated on four-point Likert Scale }\end{array}$ & $\begin{array}{l}\text { Pre-assessment } \\
\text { Post-assessment } \\
\text { Follow up }\end{array}$ \\
\hline Coping & $\begin{array}{l}\text { Children's } \\
\text { Response Style } \\
\text { Questionnaire } \\
\text { CRSQ }\end{array}$ & $\begin{array}{l}\text { Sibinga } \\
(2016)[30]\end{array}$ & $\begin{array}{l}\text { Assess coping ability by } \\
\text { measuring } 3 \text { types of } \\
\text { reactions: rumination, } \\
\text { problem solving, destruction }\end{array}$ & Self-reported survey & $\begin{array}{l}\text { Pre-assessment } \\
\text { Post-assessment }\end{array}$ \\
\hline
\end{tabular}


Table 5 Evaluation tools and methodologies used in included studies (Continued)

\begin{tabular}{|c|c|c|c|c|c|}
\hline Criteria & Tool & $\begin{array}{l}\text { First author } \\
\text { (Year) of } \\
\text { Studies in } \\
\text { which Tool } \\
\text { was Used }\end{array}$ & Purpose & Methodology & Timeframe \\
\hline & Brief COPE & $\begin{array}{l}\text { Sibinga } \\
(2016)[30]\end{array}$ & $\begin{array}{l}\text { Assess coping ability by } \\
\text { measuring } 14 \text { coping } \\
\text { approaches }\end{array}$ & Self-reported survey & $\begin{array}{l}\text { Pre-assessment } \\
\text { Post-assessment }\end{array}$ \\
\hline & $\begin{array}{l}\text { Coping Self- } \\
\text { Efficacy Scale } \\
\text { CSE }\end{array}$ & $\begin{array}{l}\text { Sibinga } \\
(2016)[30]\end{array}$ & Assess coping ability & $\begin{array}{l}\text { Self-reported survey with end } \\
\text { result of single variable }\end{array}$ & $\begin{array}{l}\text { Pre-assessment } \\
\text { Post-assessment }\end{array}$ \\
\hline & $\begin{array}{l}\text { Social Problem- } \\
\text { Solving Inventory } \\
\text { SPSI }\end{array}$ & $\begin{array}{l}\text { Kraag } \\
(2009)[39]\end{array}$ & Measure problem-solving skills & Self-reported scales & $\begin{array}{l}\text { Pre-assessment } \\
\text { Post-assessment } \\
\text { Follow up }\end{array}$ \\
\hline & $\begin{array}{l}\text { Schoolagers } \\
\text { Coping Strategies } \\
\text { Inventory }\end{array}$ & $\begin{array}{l}\text { Mishara } \\
\text { (2006) [32] } \\
\text { Dufour } \\
(2011)[34]\end{array}$ & $\begin{array}{l}\text { Gather information about } \\
\text { actual coping experiences } \\
\text { of children; identify frequencies } \\
\text { of use of coping skills }\end{array}$ & $\begin{array}{l}\text { Self-reported questionnaire, } \\
26 \text { items }\end{array}$ & $\begin{array}{l}\text { Process } \\
\text { evaluation } \\
\text { Post-assessment }\end{array}$ \\
\hline & $\begin{array}{l}\text { Draw and Write } \\
\text { Technique }\end{array}$ & $\begin{array}{l}\text { Clarke } \\
(2015)[31]\end{array}$ & $\begin{array}{l}\text { Gather personal experiences } \\
\text { of how children coped } \\
\text { with problem situations }\end{array}$ & $\begin{array}{l}\text { Part of participatory workshop: } \\
\text { children asked to draw picture } \\
\text { about emotion and draw how they coped }\end{array}$ & $\begin{array}{l}\text { Pre-assessment } \\
\text { Post-assessment }\end{array}$ \\
\hline & $\begin{array}{l}\text { KidCope } \\
\text { Questionnaire }\end{array}$ & $\begin{array}{l}\text { Holen } \\
\text { (2012) [35] }\end{array}$ & $\begin{array}{l}\text { Measure of } 10 \text { coping strategies: } \\
\text { distraction, social withdrawal, } \\
\text { cognitive restructuring, self-criticism, } \\
\text { blaming others, problem solving, } \\
\text { emotional expression, wishful thinking, } \\
\text { social support, resignation }\end{array}$ & $\begin{array}{l}\text { Self-reporting questionnaires } \\
\text { with adaptations for different } \\
\text { age groups }\end{array}$ & $\begin{array}{l}\text { Pre-assessment } \\
\text { Post-assessment }\end{array}$ \\
\hline & Focus Groups & $\begin{array}{l}\text { Malti (2008) } \\
{[36]}\end{array}$ & $\begin{array}{l}\text { Evaluation of program } \\
\text { implementation and process }\end{array}$ & $\begin{array}{l}\text { Multiple students with one } \\
\text { researcher }\end{array}$ & $\begin{array}{l}\text { Process } \\
\text { evaluation } \\
\text { Implementation } \\
\text { evaluation } \\
\text { Post-assessment }\end{array}$ \\
\hline & $\begin{array}{l}\text { Researcher- } \\
\text { developed } \\
\text { questionnaires }\end{array}$ & $\begin{array}{l}\text { Malti (2008) } \\
\text { [36] } \\
\text { Dufour } \\
(2011)[34]\end{array}$ & $\begin{array}{l}\text { Assessment of components } \\
\text { of program; assessment of } \\
\text { children's status by parents } \\
\text { and teachers }\end{array}$ & Self-reported questionnaires & $\begin{array}{l}\text { Pre-assessment } \\
\text { Process } \\
\text { evaluation } \\
\text { Post-assessment } \\
\text { Climate } \\
\text { assessment }\end{array}$ \\
\hline & Session Reports & $\begin{array}{l}\text { Mishara } \\
\text { (2006) [32] } \\
\text { Dufour } \\
\text { (2011) [34] } \\
\text { Holen } \\
\text { (2012) [35] }\end{array}$ & $\begin{array}{l}\text { Assessment of each } \\
\text { component of program }\end{array}$ & $\begin{array}{l}\text { Qualitative, self-administered } \\
\text { report by program deliverer } \\
\text { after each session }\end{array}$ & $\begin{array}{l}\text { Process } \\
\text { evaluation } \\
\text { Implementation } \\
\text { evaluation }\end{array}$ \\
\hline & Interviews & $\begin{array}{l}\text { Malti (2008) } \\
{[36]} \\
\text { Clarke } \\
(2015)[31]\end{array}$ & $\begin{array}{l}\text { Assessment of components } \\
\text { of program }\end{array}$ & $\begin{array}{l}\text { One-on-one interviews with } \\
\text { researcher or evaluator }\end{array}$ & $\begin{array}{l}\text { Pre-assessment } \\
\text { Process } \\
\text { evaluation } \\
\text { Post-assessment } \\
\text { Implementation } \\
\text { evaluation }\end{array}$ \\
\hline & $\begin{array}{l}\text { Programme } \\
\text { Fidelity Checklist }\end{array}$ & $\begin{array}{l}\text { Clarke } \\
\text { (2014) [33] } \\
\text { Caldarella } \\
\text { (2009) [39] }\end{array}$ & $\begin{array}{l}\text { Report of what portions of } \\
\text { program session fully or } \\
\text { partially implemented, } \\
\text { which ones omitted }\end{array}$ & $\begin{array}{l}\text { Self-reported checklist and } \\
\text { questionnaire }\end{array}$ & $\begin{array}{l}\text { Process } \\
\text { assessment } \\
\text { Implementation } \\
\text { evaluation }\end{array}$ \\
\hline & $\begin{array}{l}\text { Class } \\
\text { Environment } \\
\text { Climate } \\
\text { Questionnaire }\end{array}$ & $\begin{array}{l}\text { Dufour } \\
\text { (2011) [34] }\end{array}$ & $\begin{array}{l}\text { Assess climate of classroom } \\
\text { and describe teacher } \\
\text { characteristics }\end{array}$ & $\begin{array}{l}\text { Self-reported questionnaire, } 36 \\
\text { items }\end{array}$ & $\begin{array}{l}\text { Pre-assessment } \\
\text { Post-assessment }\end{array}$ \\
\hline Acceptability & $\begin{array}{l}\text { Student Self- } \\
\text { Assessment of }\end{array}$ & $\begin{array}{l}\text { Caldarella } \\
\text { (2009) [40] }\end{array}$ & $\begin{array}{l}\text { Assess student perception } \\
\text { of social validity of program }\end{array}$ & $\begin{array}{l}\text { Self-reported questionnaire, } 10 \text { questions: } \\
8 \text { with Likert Scale, } 2 \text { open ended }\end{array}$ & Post-assessment \\
\hline
\end{tabular}


Table 5 Evaluation tools and methodologies used in included studies (Continued)

\begin{tabular}{|c|c|c|c|c|c|}
\hline Criteria & Tool & $\begin{array}{l}\text { First author } \\
\text { (Year) of } \\
\text { Studies in } \\
\text { which Tool } \\
\text { was Used }\end{array}$ & Purpose & Methodology & Timeframe \\
\hline & $\begin{array}{l}\text { Intervention } \\
\text { Rating Profile-15 } \\
\text { IRP-15 }\end{array}$ & $\begin{array}{l}\text { Caldarella } \\
\text { (2009) }[40]\end{array}$ & $\begin{array}{l}\text { Assess teacher's perception } \\
\text { of social validity of program }\end{array}$ & $\begin{array}{l}\text { Self-reported questionnaire with } 15 \text { items } \\
\text { on 6-point Likert scale }\end{array}$ & Post-assessment \\
\hline
\end{tabular}

\section{Abbreviations}

AOP-PTS: Aussie optimism: positive thinking skills program; CDI: Children's depression index; MBSR: Mindfulness-Based stress reduction; MMAT: Mixed methods appraisal tool; WA: Western Australia

\section{Acknowledgements}

We thank Georgetown University, Dr. Helen Fairnie-Jones and staff at the WA Centre for Rural Health for assistance that enabled this work to be undertaken. We particularly thank May Doncon for her valuable help in early discussions and highlighting the importance of the topic.

\section{Availability of data and materials}

Data sharing is not applicable to this article as no datasets were generated or analyzed during the current study.

\section{Authors' contributions}

AF determined the search strategy, conducted the systematic review of the databases, and wrote all parts of the review. AF, ED and ST read all full text articles and agreed upon inclusion and exclusion of articles. AF and ED conducted independent applications of MMAT to the articles, and came to a consensus on article strength. ED assisted in writing the results and discussion section as well. AF and ST edited the article for content and errors. All authors read and approved the final manuscript.

\section{Ethics approval and consent to participate}

Not applicable as all literature is published.

\section{Consent for publication}

Not applicable.

\section{Competing interests}

The authors declare that they have no competing interests.

\section{Publisher's Note}

Springer Nature remains neutral with regard to jurisdictional claims in published maps and institutional affiliations.

\section{Received: 27 April 2018 Accepted: 14 June 2018}

\section{Published online: 05 July 2018}

\section{References}

1. Kaufman J, Cook A, Arny L, Jones B, Pittinsky T. Problems defining resiliency: illustrations from the study of maltreated children. Dev Psychopathol. 1994;6(1):215-29.

2. Grotberg EH: The international resilience project findings from the research and the effectiveness of interventions [microform] / Edith H. Grotberg. [Washington D.C.]: distributed by ERIC clearinghouse; 1996.

3. Greenberg M, Domitrovich C, Bumbarger B. The prevention of mental disorders in school-aged children: current state of the field. Prev Treat. 2001; 4(1):1-62 https://www.researchgate.net/profile/Brian_Bumbarger/publication/ 216458565_The_Prevention_of_Mental_Disorders_in_School-Aged_Children_ Current State of the Field/links/Ofcfd50d20aaf66cf7000000/The-Preventionof-Mental-Disorders-in-School-Aged-Children-Current-State-of-the-Field.pdf

4. Hodgson R, Abbasi T, Clarkson J. Effective mental health promotion: a literature review. Health Educ J. 1996;55(1):55-74.

5. Catalano RF, Berglund ML, Ryan JAM, Lonczak HS, Hawkins JD. Positive youth development in the United States: research findings on evaluations of positive youth development programs. ANNALS Am Acad Polit Soc Sci. 2004;591(1):98-124.
6. Benson PL, Saito RN. The Scientific Foundations of Youth Development. In: Benson PL, Pittman KJ, editors. Trends in Youth Development: Visions, Realities and Challenges. Boston, MA: Springer US; 2001. p. 135-54.

7. Rose G. Sick individuals and sick populations. Int J Epidemiol. 2001; 30(3):427-32.

8. Catherine $P B, L J$ F. Editorial commentary: resilience in child development - interconnected pathways to wellbeing. J Child Psychol Psychiatry. 2013;54(4):333-6.

9. Karatsoreos IN, McEwen BS. Annual research review: the neurobiology and physiology of resilience and adaptation across the life course. J Child Psychol Psychiatry. 2013;54(4):337-47.

10. Bonanno GA, Diminich ED. Annual research review: positive adjustment to adversity - trajectories of minimal-impact resilience and emergent resilience. J Child Psychol Psychiatry. 2013:54(4):378-401.

11. Dante C. Annual research review: resilient functioning in maltreated children - past, present, and future perspectives. J Child Psychol Psychiatry. 2013;54(4):402-22.

12. Weare K, Nind M. Mental health promotion and problem prevention in schools: what does the evidence say? Health Promot Int. 2011;26(suppl 1):i29-69.

13. Adi Y, Killoran A, Janmohamed K, Stewart-Brown S. Systematic Review of the Effectiveness of Interventions to Promote Mental Wellbeing in Primary Schools: Universal Approaches Which Do not Focus on Violence or Bullying. London: National Institute for Clinical Excellence; 2007.

14. Diekstra R. Effectiveness of school-based social and emotional education programmes worlwide - part one, a review of meta-analytic literature. In: Social and motional education: an international analysis edn.: Fundacion Marcelino Botin; 2008. p. 255-84.

15. Browne G, Gafni A, Roberts J, Byrne C, Majumdar B. Effective/efficient mental health programs for school-age children: a synthesis of reviews. Soc Sci Med. 2004:58(7):1367-84.

16. Durlak JA, Weissberg RP, Dymnicki AB, Taylor RD, Schellinger KB. The impact of enhancing students' social and emotional learning: a metaanalysis of school-based universal interventions. Child Dev. 2011;82(1): 405-32.

17. Murphy JM, Abel MR, Hoover S, Jellinek M, Fazel M. Scope, scale, and dose of the world's largest school-based mental health programs. Harv Rev Psychiatry. 2017;25(5):218-28.

18. Dray J, Bowman J, Campbell E, Freund M, Wolfenden L, Hodder RK, McElwaine K, Tremain D, Bartlem K, Bailey J, et al. Systematic review of universal resilience-focused interventions targeting child and adolescent mental health in the school setting. J Am Acad Child Adolesc Psychiatry. 2017:56(10):813-24.

19. Wells J, Barlow J, Stewart-Brown S. A systematic review of universal approaches to mental health promotion in schools. Health Educ. 2003;103(4):197-220.

20. Durlak JA, Wells AM. Primary prevention mental health programs for children and adolescents: a meta-analytic review. Am J Community Psychol. 1997:25(2):115-52.

21. Zoritch B, Roberts I, Oakley A. The health and welfare effects of daycare: a systematic review of randomised controlled trials. Soc Sci Med (1982). 1998;47(3):317-27

22. Gillham JE, Reivich KJ, Freres DR, Chaplin TM, Shatté AJ, Samuels B, Elkon AGL, Litzinger S, Lascher M, Gallop R, et al. School-based prevention of depressive symptoms: a randomized controlled study of the effectiveness and specificity of the Penn resiliency program. J Consult Clin Psychol. 2007;75(1):9-19.

23. Vuijk P, van Lier PA, Crijnen AA, Huizink AC. Testing sex-specific pathways from peer victimization to anxiety and depression in early adolescents through a randomized intervention trial. J Affect Disord. 2007;100(1-3):221-6. 
24. Dadds MR, Roth JH. Prevention of anxiety disorders: results of a universal trial with young children. J Child Fam Stud. 2008;17(3):320-35.

25. Sawyer MG, Pfeiffer S, Spence SH, Bond L, Graetz B, Kay D, Patton G, Sheffield J. School-based prevention of depression: a randomised controlled study of the beyondblue schools research initiative. J Child Psychol Psychiatry. 2010;51(2):199-209.

26. lizuka CA, Barrett PM, Gillies R, Cook CR, Marinovic W. A combined intervention targeting both Teachers' and Students' social-emotional skills: preliminary evaluation of Students' outcomes. Aust I Guid Couns. 2014; 24(2):152-66.

27. Rooney R, Hassan S, Kane R, Roberts CM, Nesa M. Reducing depression in 910 year old children in low SES schools: a longitudinal universal randomized controlled trial. Behav Res Ther. 2013;51(12):845-54.

28. Pluye P, Robert E, Cargo M, Bartlett G, O'Cathain A, Griffiths F, Boardman F, Gagnon MP, Rousseau MC. Proposal: A mixed methods appraisal tool for systematic mixed studies reviews. Montreal, Canada: McGill University; 2011.

29. National Collaborating Center for Methods and Tools. Appraising Qualitative, Quantitative, and Mixed Methods Studies included in Mixed Studies Reviews: The MMAT. Hamilton, ON: McMaster University; 2015.

30. Sibinga EMS, Webb L, Ghazarian SR, Ellen JM: School-based mindfulness instruction: an RCT. Pediatrics 2016, 137 (1) (no pagination)(e20152532).

31. Clarke AM, Sixsmith J, Barry MM. Evaluating the implementation of an emotional wellbeing programme for primary school children using participatory approaches. Health Educ J. 2015;74(5):578-93.

32. Mishara BL, Ystgaard M. Effectiveness of a mental health promotion program to improve coping skills in young children: Zippy's friends. Early Child Res Q. 2006;21 (1):110-23.

33. Clarke AM, Bunting B, Barry MM. Evaluating the implementation of a schoolbased emotional well-being programme: a cluster randomized controlled trial of Zippy's friends for children in disadvantaged primary schools. Health Educ Res. 2014;29(5):786-98.

34. Dufour S, Denoncourt J, Mishara BL. Improving Children's adaptation: new evidence regarding the effectiveness of Zippy's friends, a school mental health promotion program. Adv School Ment Health Promot. 2011;4(3):18-28.

35. Holen S, Waaktaar T, Lervåg A, Ystgaard M. The effectiveness of a universal school-based programme on coping and mental health: a randomised, controlled study of Zippy's friends. Educ Psychol. 2012;32(5):657-77.

36. Malti T, Schwartz SE, Liu CH, Noam GG. Program evaluation: relationships as key to student development. New Dir Youth Dev. 2008;2008(120):151-77. Table of Contents

37. Nielsen L, Meilstrup C, Nelausen MK, Koushede V, Holstein BE. Promotion of social and emotional competence. Health Educ. 2015;115(3/4):339-56.

38. Yamamoto T, Matsumoto Y, Bernard ME. Effects of the cognitive-behavioral you can do it! Education program on the resilience of Japanese elementary school students: a preliminary investigation. Int J Educ Res. 2017;86:50-8.

39. Kraag G, Van Breukelen GJP, Kok G, Hosman C. Learn young, learn fair', a stress management program for fifth and sixth graders: longitudinal results from an experimental study. J Child Psychol Psychiatry. 2009:50(9):1185-95.

40. Caldarella P, Christensen L, Kramer TJ, Kronmiller K. Promoting social and emotional learning in second grade students: a study of the strong start curriculum. Early Childhood Educ J. 2009;37(1):51-6.

41. Durlak JA, DuPre EP. Implementation matters: a review of research on the influence of implementation on program outcomes and the factors affecting implementation. Am J Community Psychol. 2008;41(3-4):327-50.

42. Moore, TG. Rethinking universal and targeted services. CCCH Working Paper 2. August 2008. Parkville, Victoria: Centre for Community Child Health.

43. Darbyshire P, MacDougall C, Schiller W. Multiple methods in qualitative research with children: more insight or just more? Qual Res. 2005;5(4):417-36.

44. Pridmore P, Bendelow G. Images of health: exploring beliefs of children using the 'draw-and-write' technique. Health Educ J. 1995;54(4):473-88.

45. Barter $\mathrm{C}$, Renold E. 'I wanna tell you a story': exploring the application of vignettes in qualitative research with children and young people. Int J Soc Res Methodol. 2000;3(4):307-23.

46. Lengua LJ, Stormshak EA. Gender, Gender Roles, and Personality: gender differences in the prediction of coping and psychological symptoms. Sex Roles. 2000;43(11):787-820

47. Wasonga T, Christman DE, Kilmer L. Ethnicity, Gender and Age: predicting resilience and academic achievement among urban high school students. Am Second Educ. 2003;32(1):62-74.

\section{Ready to submit your research? Choose BMC and benefit from:}

- fast, convenient online submission

- thorough peer review by experienced researchers in your field

- rapid publication on acceptance

- support for research data, including large and complex data types

- gold Open Access which fosters wider collaboration and increased citations

- maximum visibility for your research: over $100 \mathrm{M}$ website views per year

At BMC, research is always in progress.

Learn more biomedcentral.com/submissions 